\title{
Automatic testing of cognitive performance in baboons maintained in social groups
}

\author{
Joël Fagot and Dany Paleressompoulle \\ Université d'Aix-Marseille, Marseille, France
}

\begin{abstract}
Laboratory procedures used to study the cognitive functions of primates traditionally have involved removal of the subjects from their living quarters to be tested singly in a remote experimental room. This article presents an alternative research strategy favoring testing primates while they are maintained in their social group. The automatic learning device for monkeys (ALDM) is a computerized test system controlled by an automatic radio frequency identification of subjects. It is provided ad lib inside the social group of monkeys, for voluntary self-testing on a 24-h schedule. Nine baboons were tested with ALDM during a 7-month period. Experiments were performed to assess learning in motor control and abstract reasoning tasks. The results revealed high trial frequencies and excellent learning performance, even in tasks involving the highest cognitive complexities. A different study using ALDM with a group of 3 rhesus monkeys revealed social influences on learning. Beyond its interest for cognitive psychologists, ALDM is of interest for pharmacologists and cognitive neuroscientists working with nonhuman primates. ALDM also can serve as an enrichment tool for captive animals and may be used to study a variety of species other than primates.
\end{abstract}

With very few counterexamples (Andrews \& Rosenblum, 1994; Fagot, Drea, \& Wallen, 1991), researchers interested in primate nonsocial cognition have favored the test of isolated animals (for a review, see Drea, 2006). In these studies, the primates have traditionally been caught for their daily testing in an experimental area spatially separated from their living quarters. This classical procedure (CP) has demonstrated its efficiency. Most of what is currently known on primate cognition-for instance, regarding their perception, memory, reasoning, and conceptualization - has been collected in that way. The CP has also regularly been used in cognitive neuroscience studies involving nonhuman primates.

In spite of its efficiency, the CP has many important limitations calling for alternative experimental procedures for testing primates in laboratories. One first set of concerns comes from the domain of ethics. Primates are gregarious animals (Silk, 1984). A great deal of their activity in natural settings is devoted to social interactions (Altman \& Altman, 1971). Depriving primates of social activities has deleterious effects on their well-being (Griffin \& Harlow, 1966). Forced separations from housing partners for daily testing appear to be potential sources of stress that may affect well-being and, as a consequence, the general significance of scientific results collected on these animals. Beyond these ethical concerns, $\mathrm{CP}$ also has serious practical drawbacks. Catching procedures are risky because of potential injuries, bites, or transmitted diseases. The mandatory presence of a technician during experiments is time consuming and costly. The $\mathrm{CP}$ therefore seriously limits the number of subjects tested per day or the number of trials that can be proposed for each primate in a test session. In a more scientific perspective, the $\mathrm{CP}$ also limits the range of topics that can be assessed in laboratories - in particular, those topics related to social cognition. The field of primate cognition appears to be structured as two relatively independent subfields, with little interaction between them. These are the subfield bearing on the understanding of the physical world by primates, in which the CP is mainly used, and the field of social cognition, in which more ethological approaches involving animals in social groups are used. This apparent split between social and nonsocial studies derives from the extreme difficulty involved in inferring social understanding from the broadly used $\mathrm{CP}$ procedure.

This article presents a new experimental procedure whose aim is to alleviate the above ethical, practical, and scientific concerns. This methodology was inspired by early attempts to conduct cognitive tests in captive social groups of nonhuman primates (Andrews \& Rosenblum, 1994; Drea, 2006; Fagot et al., 1991). These early experiments showed that monkeys who received cognitive tests in social contexts could occasionally develop sophisticated social strategies, such as playing dumb (Drea \& Wallen, 1999). However, learning in these experiments has appeared thus far to be limited to relatively simple tasks involving, for instance, the discrimination of baited boxes of different colors (Fagot et al., 1991) or the use of a joystick to select a target on a screen (Andrews \& Rosenblum, 1994). Evans, Beran, Chan, Klein, and Menzel (2008) recently developed a procedure for training capuchin monkeys to be voluntarily isolated and confined in a test room connected to their living quar- 
ters. This procedure alleviates potential stresses due to the capture but still requires the presence of someone to isolate and release the subjects.

We recently developed a new experimental procedure that complements the above attempts to study primates in groups. This system has, in our perspective, four main advantages: (1) It allows testing of the subjects in their social group; (2) it makes the presence of the experimenter unnecessary because of the automaticity of the protocol; (3) it allows data recording at a high frequency and, moreover, on a 24-h schedule; and finally, (4) it permits testing of complex social and nonsocial cognitive skills, with sophisticated experimental designs and measurements. The aim of this article is to present our new automatic learning device for monkeys (ALDM) and to offer a first set of data showing the efficiency of the method for studying a broad range of cognitive processes.

\section{METHOD}

\section{Subjects and Housing}

The subjects were nine 22-year-old Guinea baboons (Papio papio) living in three social groups of 4 males (M03, M05, M07, and M09), 3 females (F04, F06, and F08), and 2 males (M11 and M15) within the C.N.R.S. facility. Each group was housed within a $6 \times 3 \mathrm{~m}$ indoor enclosure connected to an outdoor enclosure of the same size $\left(36 \mathrm{~m}^{2}\right.$ altogether). All indoor enclosures contained sleeping niches, a tunnel in which the subjects could be sequestered during the cleaning period, various structures for climbing, and a dispenser for water. Each outdoor enclosure contained more climbing structures and an automatic test system, described below.

All the baboons had a long experimental history. They had already been tested in a variety of computerized tasks involving screens for stimulus presentation and a joystick for responding (Fagot \& Cook, 2006; Fagot \& Deruelle, 1997). They were, however, fully naive with regard to touch screens at the beginning of the research. The baboons were never food deprived. They received their daily ration of food (fruits, vegetables, and monkey chows) once a day, between 9 a.m. and 4 p.m., and every day were sequestered inside the indoor tunnel for approximately $1 \mathrm{~h}$ for cleaning purposes. M03 and M09 continued to participate in experiments with a different joystick-controlled computerized test system at the rate of approximately $1-2 \mathrm{~h} /$ day while being ALMD tested during the remainder of the day.

\section{General Principles of ALDM}

ALDM is a computerized test system permanently installed in each of the three outdoor enclosures for the baboons. Each test system comprises a device for automatic identity recording, a touch screen for stimulus presentation, a touch pad for hand response recording, and a dispenser for reward delivery, in addition to an Internet connection for a remote control for the test software. To have the best possible control of the experimental variables - for instance, in terms of viewing distance - the test equipment is concealed inside a freely accessible test chamber with an open entrance on its back side. Because ALDM is active on a 24-h sched- ule, baboons can voluntarily enter the chamber whenever it is empty, for self-testing. The computer program collects identification signals from microchips injected in the forearm of each baboon. Identity signals serve as input to control the individual experimental regimen of the subjects and to record relevant subject, trial, and response parameters.

Figure 1A shows a schematic view of a test chamber equipped with the ALDM test system. The test chamber consists of a box mounted on four 1-m-high piles. The innermost side of the test chamber, opposite to the fully opened entrance, is fitted with a view port and two hand ports (see Figure 1B). Looking through the view port allows vision of a 17 -in. touch screen, $25 \mathrm{~cm}$ from the view port and parallel to it, which can be touched by inserting one hand through one hand port. Two antennas fixed around the hand ports allow identification of the animal inside the test booth once it has inserted one forearm (and thus, one microchip) into one of the two hand ports. Either hand can be used for self-identification.

A large box adjacent to the test chamber contains the touch screen and the controlling computer. This box is installed outside the outdoor enclosure, along the wire mesh, whereas the test chamber facing it is installed inside the enclosure (Figure 1C). Equipment other than the screen is concealed from view and is inaccessible to the monkeys. Use of an opaque waterproof Perspex material to build the test chamber allows presentation of the stimuli in well-standardized conditions, irrespective of weather and lighting fluctuations. Food reward is delivered inside the test chamber, in a receptacle fixed on the bottom part of its inner front side.

\section{ALDM System Components}

Identification device. Biocompatible $1.2 \times 0.2 \mathrm{~cm}$ radio frequency identification (RFID) microchips were used (Reseaumatique; Bernay, France). RFID signals from the microchips were captured by two homemade antennas mounted in parallel and connected to a radio identification microchip reader (model Petscan RT100 Version 5, Real Trace; Villebon sur Yvette, France). Identity signals were provided to the computer through the serial port.

Computer and touch monitor. The experiment was controlled by a precision 670 Dell 3.2-GHz PC computer running with Microsoft Windows XP, which was connected through a USB port to an LCD capacitive touch screen (model ET1739L from Elotouch; Berwyn, IL). That model of touch monitor was selected because of its size (17 in.) and its built-in 3-mm-wide secure glass protecting the screen. The computer was equipped with speakers to deliver auditory signals.

Dispenser and rewards. ALDM allows a very large number of trials to be collected per individual/day and thus requires the use of a highly appreciated but relatively inexpensive reward. Ebly dry wheat (www.ebly.co.uk/an/ indexfra.htm) was used as rewards because it satisfied these requirements. Rewards were delivered inside the test chamber by a pipe connected to a homemade dispenser. That dispenser consisted of a motorized cylinder drilled with a small hole that made a complete turn to deliver by gravity a small amount of 1-3 grains on each rewarded 


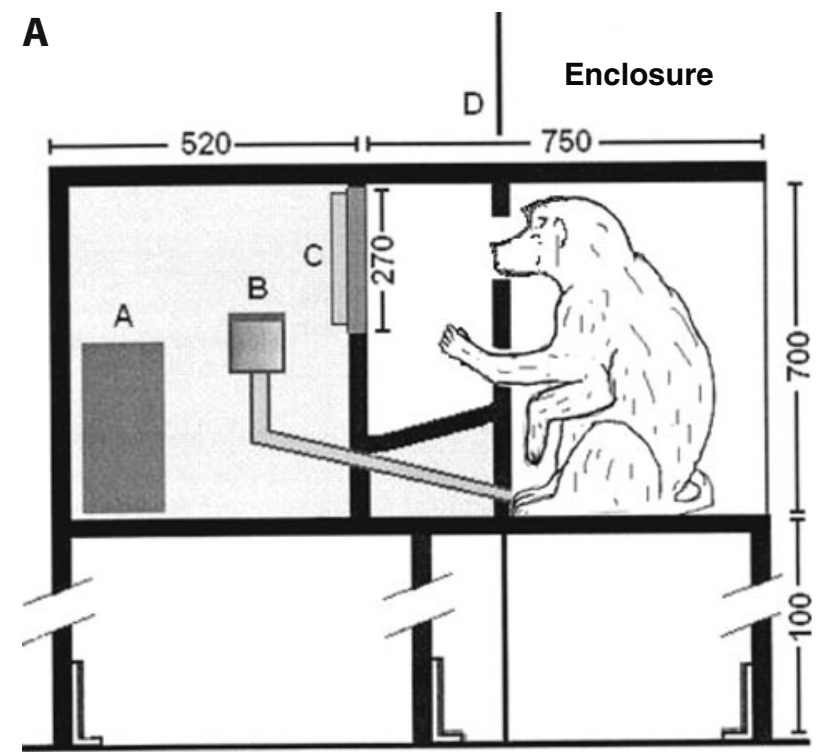

B

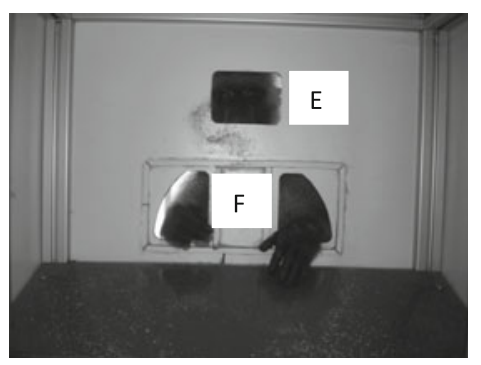

C

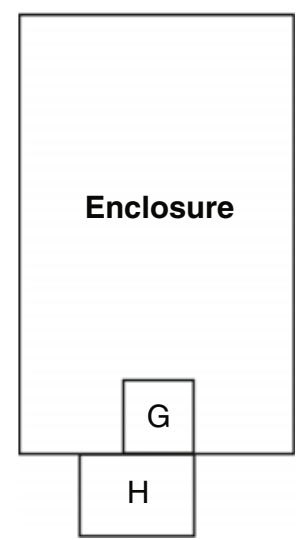

Figure 1. Schematic description of ALDM. (A) Test chamber as shown laterally from its left side. (B) A baboon watching the screen through the viewport. (C) Bird's-eye view of the enclosure and test chamber. $A$, computer; $B$, food dispenser; $C$, 17-in. touch monitor; $D$, fence of the enclosure; $E$, view port allowing vision of the screen; $F$, hand port with surrounding antennas for identity detection; $G$, test chamber; $H$, compartment concealing the outof-reach computer, food dispenser, and other hardware devices. Climbing inside the test chamber was facilitated by a ladder not visible in this graph.

trial. The command signals were delivered to the dispenser through the parallel port.

Internet remote control of the experiment. Internet remote control was made possible by an Access Remote PC (www.access-remote-pc.com/). This feature of the test system allows remote supervision of the research and remote interaction with the test program - for instance, to launch or stop it.

Computer software. The experiment was controlled by a test program developed by the first author with EPrime (Version 1.2, Psychology Software Tools, Pittsburgh). With this program, each subject receives its test- ing protocol at its own rhythm, irrespective of the order in which the members of its group entered the test chamber. E-Prime provides a large number of routines for displaying the stimuli and recording response times (RTs). However, because E-Prime has no built-in options to test several subjects in parallel, scripts were added to the program to upload the relevant subject's information at the beginning of each trial and to update and save that information immediately after the trial had been run.

Figure 2 represents the flowchart of the test program. The program was structured in three phases: the training, test, and standby phases. A trial in each phase was initiated when one arm was introduced into one of the two arm ports for self-identification. The training phase consisted of series of training trials (hereafter named sessions, although trials within a session were not necessarily run in succession, due to subject changes), which were repeated until a predefined learning criterion had been reached ( $80 \%$ correct in two consecutive sessions in our research). The test phase implied the repetition of randomly ordered test sessions until a predefined number of test sessions had been run. Finally, the standby phase consisted of an easy two-alternative forced choice discrimination task, in which the subject had to select the red square from two squares on the screen. That standby phase continued until the experimenter interrupted the test program, after all the subjects in the group had gone through the experimental phases. The standby phase had no real scientific interest. It served only to keep the faster baboons active while the slowest animals were catching up. The exact content of the training and test trials was flexibly altered from one experiment to the next, using E-Prime built-in options.

Two other important features were added to the program to facilitate learning. They were the possible use of different reinforcement regimen (e.g., extinction and differential or random reinforcement at a predefined rate) and the optional use of a correction procedure in which an erroneous trial could be represented a fixed number of times, defined by the experimenter (a maximum of three correction trials was used in our research), until a correct response was made. A session time-out of 15-30 min was systematically imposed on each subject after completion of a session. During that time-out period, the trials were no longer triggered by the identity signal. Since only the subject(s) who had just finished a session were timed out, use of the time-out procedure promoted a turnover inside the test chamber. That time-out procedure was efficient in limiting conflicts between individuals from the same group, probably because all the subjects understood that they would be tested in turn. In practice, informal observations revealed only a few conflicts during the first 2 weeks after ALDM had been introduced to the groups, but these conflicts quickly disappeared with continued testing.

Sessions consisted of 92-148 randomly ordered trials, depending on the exact training or test protocol. In our tasks, correct trials delivered 1-3 grains of dry wheat and a tone. Incorrect responses gave rise to a 3 -sec green screen serving as trial time-out and a different auditory signal of lower frequency. There was no confirmation of subject identity after the beginning of each trial. However, trials with RTs greater 


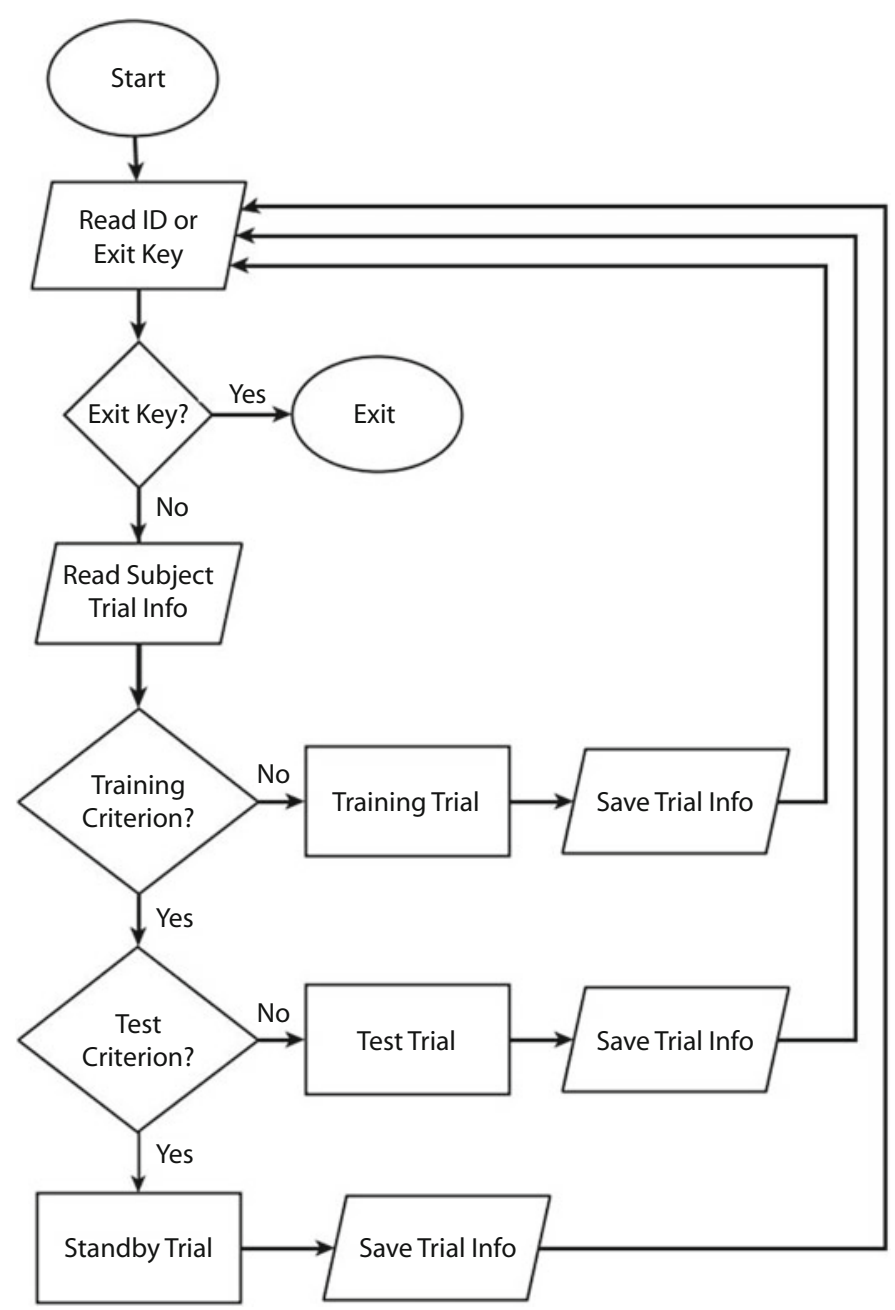

Figure 2. Flowchart of the main structure of the test software.

than $15 \mathrm{sec}$ were systematically neglected for data recording, and thus re-presented, to prevent consideration of the trials initiated by 1 subject and finished by another one.

Recorded variables. Table 1 shows data recording information relevant to understanding the test process (variables describing the actual trial-for instance, stimulus identity or its location on the screen - are omitted). Table 1 illustrates a behavioral sequence in which 3 baboons interacted with ALDM. An inspection of the trial numbers reveals that the program systematically resumed testing each individual at its previous state, after a break that potentially was due to the testing of another subject. Because of that procedure, each baboon could be tested independently with its full experimental design, with no lost trials, whatever the order in which the subjects entered the test chambers.

\section{RESULTS}

ALDM systems were provided to the three groups of baboons during the autumn of 2007. Reported below are preliminary data collected from January to July 2008 - that is, after the baboons have been habituated to the test systems. One male baboon (i.e., M09) died during the test period for a reason unrelated to the research protocol (septicemia). Only 2 months of recording will thus be reported for this monkey. Several experiments were run during that 7 -month time period, including experiments on motor control or reasoning. While the data provided below is examined, it should be kept in mind that some of the baboons continued to be tested with the $\mathrm{CP}$ joystick-controlled procedures during the study period, in parallel to the tests run with ALDM. ALDM systems were also occasionally stopped during several days for maintenance or programming, further limiting the number of trials recorded during that period.

\section{Trial Frequencies}

A very large number $(702,021)$ of trials were recorded during the 7 months of the experiment. Figure 3 reports how these trials were distributed across individuals. The automatic test system proved highly efficient for testing almost every subject, since all the baboons but 1 (i.e., F04) interacted with the test system at very high frequencies. Excluding the latter inactive baboon, the average frequen- 
Table 1

Sample of Data Recording

\begin{tabular}{cccccccc}
\hline Name & Experiment & Phase & Date & Time & Trial & Score & Correction \\
\hline 5 & RMTS & training & $11 / 08 / 07$ & $07: 57: 43$ & 16 & 1 & NO \\
5 & RMTS & training & $11 / 08 / 07$ & $07: 58: 01$ & 17 & 0 & NO \\
5 & RMTS & training & $11 / 08 / 07$ & $07: 58: 17$ & 17 & 1 & YES \\
5 & RMTS & training & $11 / 08 / 07$ & $07: 58: 43$ & 18 & 1 & NO \\
5 & RMTS & training & $11 / 08 / 07$ & $07: 59: 05$ & 19 & 0 & NO \\
7 & RMTS & training & $11 / 08 / 07$ & $07: 59: 56$ & 1 & 1 & NO \\
7 & RMTS & training & $11 / 08 / 07$ & $08: 00: 11$ & 2 & 0 & NO \\
7 & RMTS & training & $11 / 08 / 07$ & $08: 00: 27$ & 2 & 1 & YES \\
5 & RMTS & training & $11 / 08 / 07$ & $08: 02: 29$ & 19 & 1 & YES \\
5 & RMTS & training & $11 / 08 / 07$ & $08: 02: 45$ & 20 & 1 & NO \\
5 & RMTS & training & $11 / 08 / 07$ & $08: 03: 00$ & 21 & 1 & NO \\
9 & RMTS & training & $11 / 08 / 07$ & $08: 09: 07$ & 1 & 1 & NO \\
9 & RMTS & training & $11 / 08 / 07$ & $08: 09: 21$ & 2 & 1 & NO \\
\hline
\end{tabular}

Note-Columns indicate (from left to right) the subject's name, the name of the experiment, the experimental phase, the trial date, the trial time, the trial number within the series, the score $(1=$ correct, $0=$ incorrect $)$, and whether the trial was a correction trial or not. RMTS, relational matching-to-sample problem.

cies of trials obtained per animal ranged from 298 to 774 $(S D=146)$ per day of testing, but some baboons could occasionally perform up to 1,800 trials a day. F04's behavior with the testing system (120 interactions over the 7-month period) can be explained by the high dominance of F06, which inhibited her behavior.

The number of consecutive trials done on average in each behavioral sequence was computed for each animal. Neglecting possible breaks induced by the session timeout, the baboons did an average of 80.7 consecutive trials in each bout (range, 32.5-284.5; $S D=99.2$ ). This propensity to work for long bouts provides appropriate conditions for learning.

We also verified the distribution of the trials, on average, for the 8 active baboons (F04 excluded), depending on test month (from January to July) and time of day (from 6 a.m. to 5 a.m. next day; see Figure 4). The test systems were occupied almost full-time from 6 a.m. to 18 p.m. There were, however, seasonal variations, since most of the activity occurred during the winter from 8 a.m. to 16 p.m., whereas the activity was more widely distributed from 6 a.m. to 22 p.m. during the summer. Amazingly, 5.1\% of the trials occurred during the night (between 11 p.m. and 5 a.m.) in June and July (see Figure 4). This activity at night reveals an extremely high motivation to work, considering (1) that the baboons were not food deprived and (2) that the test systems were installed in an outdoor enclosure with no lighting, which must be frightening for these diurnal monkeys. The two groups of males had relatively pacified relationships, although agonistic interactions occasionally occurred. All the animals in these two groups showed frequent shifts inside the test systems during the day. The

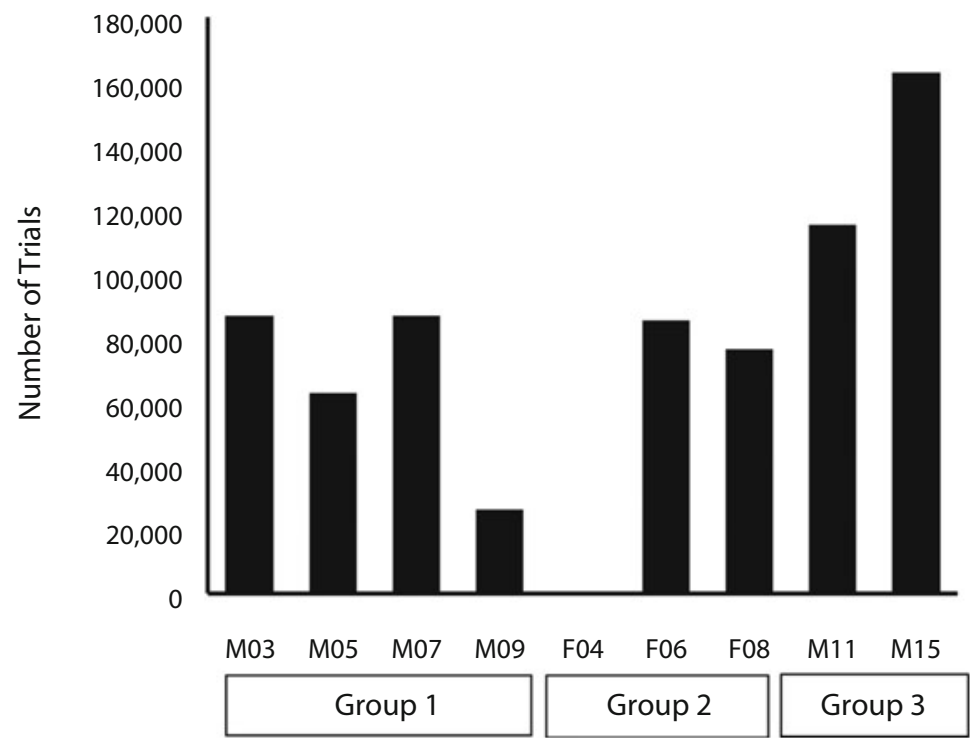

Figure 3. Frequencies of trials recorded per baboon. Note that M09 could be recorded only for $\mathbf{2}$ months, explaining the reduced number of trials for this animal. The others were recorded during a period of 7 months. 


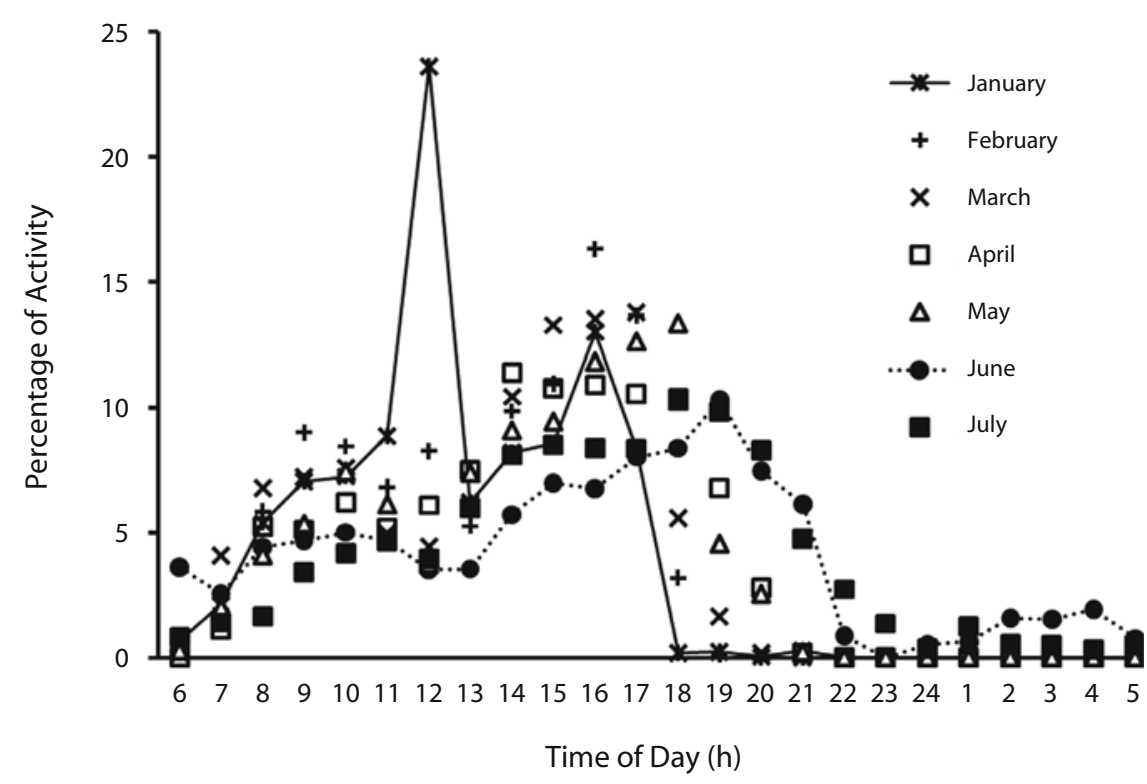

Figure 4. Distribution of the trials as a function of test month (from January to July 2008) and time of day. Distributions for the shortest (January) and longest (June) days are indicated by connecting lines.

social structure was different for the group of 3 females, since 1 of them (i.e., F06) exerted a strong dominance over her two social partners. That dominance explains why B04 showed virtually no interaction with the test system. It remains unclear whether F04 inhibited herself or was forbidden access to the test system. Figure 5 plots the temporal distribution of the activity by the 2 active females of the group. This graph shows that F06 was active during the daytime and that F08 was active during the evening and night. In spite of these variations in schedules, the 2 baboons could achieve an approximately equal amount of trials during the test period (see Figure 3). Baboons can thus flexibly adapt their activity inside ALDM to accommodate social constraints.

Finally, a correlation was computed between the number of trials performed on each testing day and the outcome of these trials in terms of reward. For each baboon, trial frequencies and percentages of reward delivery were computed separately, and the findings obtained for all the subjects (except F04) were pooled together to compute a correlation at the group level. These two variables correlated positively (see Figure 6), showing that the baboons tended to work more when they had a high rate of success (and thus of rewards) in our tasks.

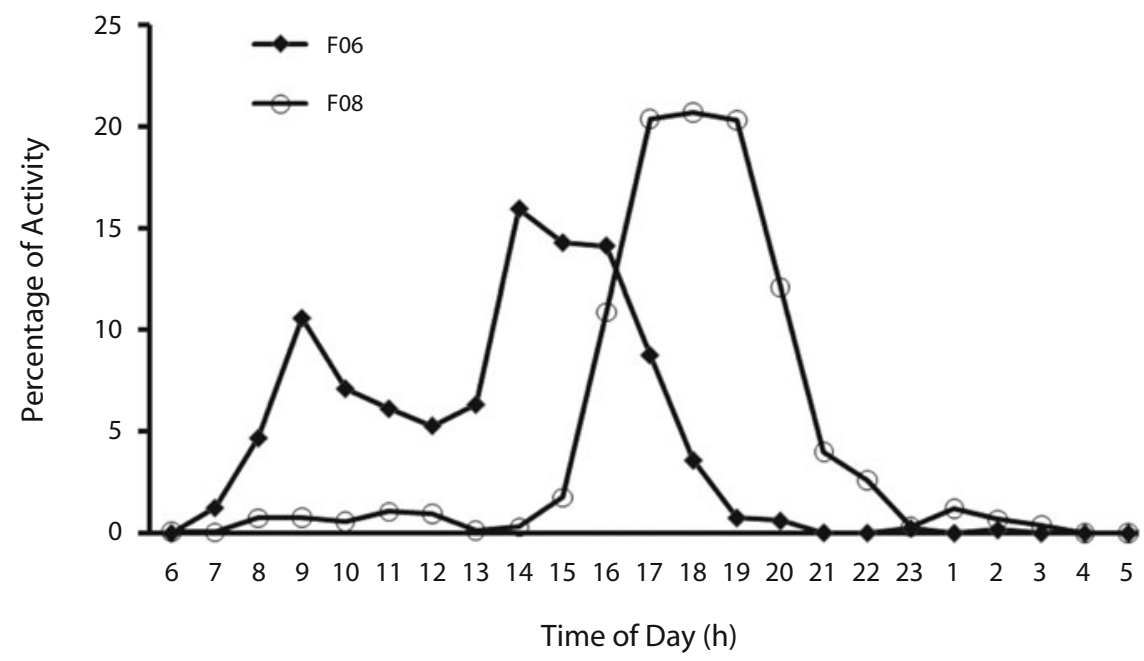

Figure 5. Time-of-day distribution of the trials for F06 (the dominant female in Group 2) and $\mathbf{F 0 8}$ (a subordinate female). Note the shift in the two distributions, suggesting an adaptation to social constraints. 


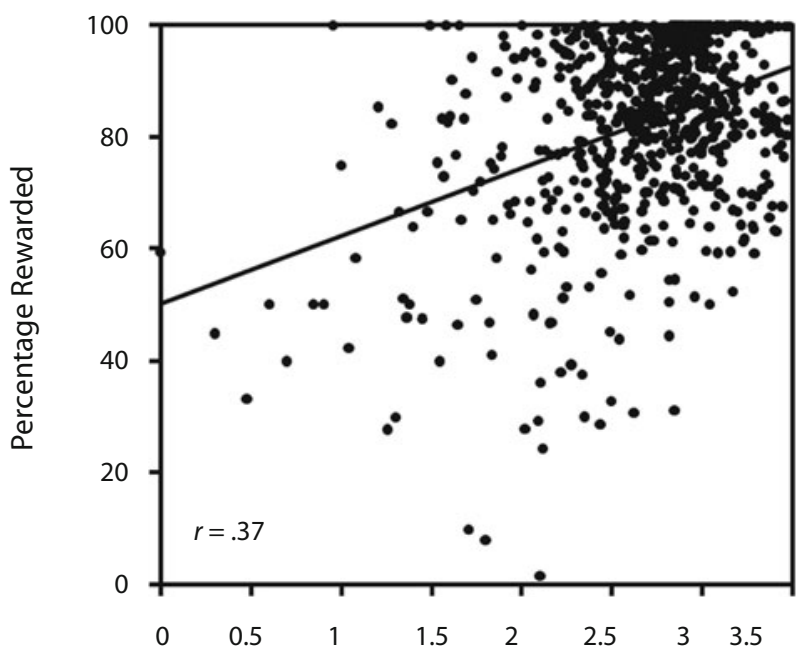

Number of Trials/Day (Log Transformed)

Figure 6. Illustration of the correlation between the frequency of trials performed in a day and reward delivery during these trials. Trial frequencies were log transformed because of potential ceiling effects at high trial frequencies. The correlation, as computed by a Pearson coefficient test, is indicated by $r$.

In brief, trial frequencies revealed an extremely high motivation for the baboons for self-testing. Beyond this already important finding, it remains to be demonstrated that baboons can learn complex tasks and, therefore, that ALDM can be used to assess a broad range of psychological phenomena. To achieve this goal, we will report below a subset of results obtained during the 7-month period described above. Complementary information can be found in Fagot, Bonté, and Parron (2008), presenting another experiment on picture perception run with the baboons during that period. We also will briefly report the results of another experiment on social influences on learning, run on a small group of rhesus monkeys.

\section{Study 1: Motor Control}

To confirm the reliability of RT measurements, Study 1 was performed to assess whether responses recorded with the ALDM can be predicted by laws derived from human motor control studies. In human-computer interaction and ergonomics, the time required to rapidly point to a target obeys Fitts's (1954) law. Taking into account target size and target distance, Fitts's law allows computation of an index of task difficulty (ID), which in humans correlates positively with RTs (Fitts, 1954). In our experiment, baboons M11 and M15 were trained to alternately point to two squares on the screen to obtain a reward. After several thousand training trials, they were tested with random variations in target sizes (from 6 to 200 pixels) and target separations (from 112 to 600 pixels). For the 2 baboons, RT was a direct function of Fitts's ID. The correlations were of .84 for M11 and .89 for M15, showing that Fitts's law can be replicated by ALDM testing.

During the next testing phases, we investigated the ability of the monkeys to actively control the difficulty of the task. Two interactive keys were introduced into the display, one at its bottom right and one at its bottom left. On these new trials, task difficulty increased during testing as target size decreased and target distance increased. However, the baboons could use the target key of their choice to actively alter the difficulty of the task. Hence, touching the bottom right interactive key randomly changed target size, whereas touching the bottom left key changed target distance. Figure 7 shows the number of trials (over 6,000 per baboon) for which actions on the size key either reduced (ID difference below 0 ) or increased (ID difference above 0) task difficulty. Interestingly, the baboons quickly learned to use the keys until ID was reduced, and they did so proactively, at the first display of the difficult trials. Use of these keys suggests that the baboons could judge the difficulty of the task and were aware of it, an ability that might be a source of metacognition.

\section{Study 2: Abstract Reasoning}

This experiment (Fagot \& Parron, 2009) employed ALDM to test the ability of monkeys to solve relational matching-to-sample (RMTS) problems. RMTS tasks imply that the subjects first judge whether a first pair of stimuli is composed of two identical or two different items and then match that pair with another comparison pair showing the same (same or different) abstract relation as the sample pair. It has been claimed that responsiveness to a relation between relations is possible only in language- or symboltrained apes (Premack, 1983; Thompson, Oden, \& Boysen, 1997). In our study, 6 baboons first viewed and touched a sample stimulus made of two adjacent $100 \times 200$ pixel rectangles of either the same (same relation) or different (different relation) colors. They were then presented with two new pairs of same and different color patches, all constructed with colors different from those of the sample pair. Selecting the stimulus pair instantiating the same relation as that in the sample was rewarded. All baboons but 1 (M07)

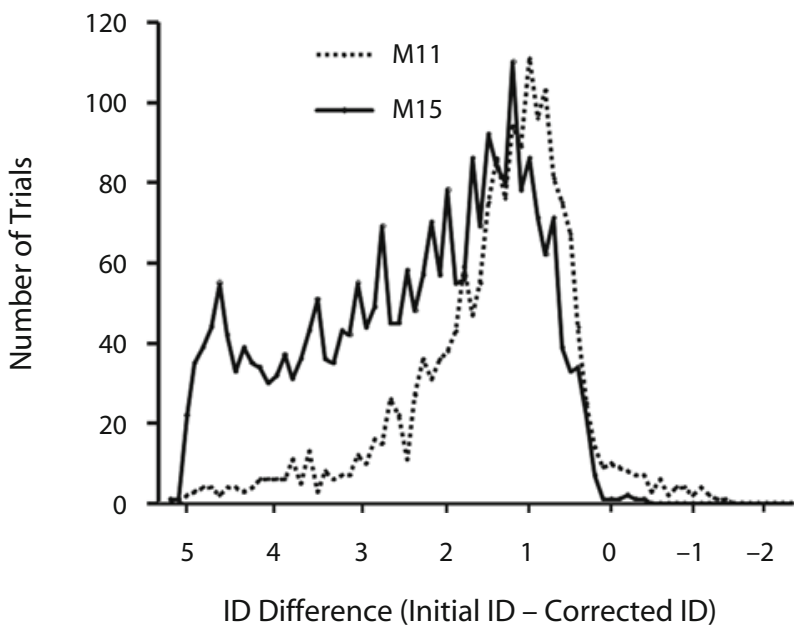

Figure 7. Number of trials for which actions on the size key either reduced (ID difference above 0 ) or increased (ID difference below 0 ) task difficulty. Note that both baboons could accurately use the size key to reduce the difficulty of the task. 


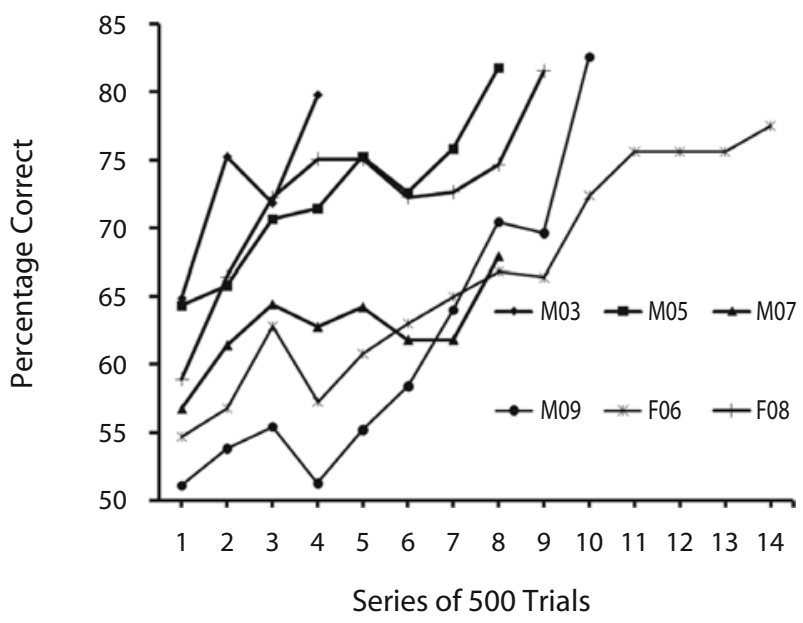

Figure 8. Learning curves in Study 2 for relational matchingto-sample performance. The data were computed after correction trials had been discarded.

learned the task to a criterion of $80 \%$ correct in two consecutive sessions (see Figure 8). Postlearning tests showed that performance deteriorated when a gap was introduced between the color patches but that this difficulty could be overcome with training. Demonstration of RMTS abilities confirms that ALDM can be a very efficient tool for assessing the highest cognitive functions of monkeys (for additional details, see Fagot \& Parron, 2009).

\section{Study 3: Social Influences on Learning}

The aim of Study 3 was to demonstrate the usefulness of ALDM for assessing social aspects of learning. It furthermore illustrates possible uses of ALDM in pharmacological researches. Study 3 involved three group-raised rhesus monkeys, comprising one 17-year-old alpha male (Roman), one 21-year-old female adult (Elisabeth), and their 1-year-old daughter (Alison). These animals were naive with respect to computerized screens prior to testing. The test system was an earlier prototype of ALMD that followed the same principles as above but allowed manipulation of the screen when the animals sat on the floor. In addition, instead of running on a $24-\mathrm{h}$ schedule, the test was made available during sessions of $2 \mathrm{~h}$ per day.

Study 3 used the well-known transfer index task (Rumbaugh, 1970), in which the positive stimulus on the screen had to be discriminated from the negative one until a criterion of either $64 \%$ or $84 \%$ correct was reached (for details on the procedure, see Rumbaugh, 1970). The rhesus monkeys received a food reward when they touched the positive $(\mathrm{S}+)$ stimulus. Reinforcement contingencies were changed during the next 11 postlearning trials, $\mathrm{S}+$ becoming $\mathrm{S}-$ and vice versa. In a first training phase that lasted 2 weeks, the subjects were requested only to learn a pair of stimuli with normal reinforcement contingencies. ALDM was initially available full time, but availability was progressively limited to $2 \mathrm{~h}$ per day. Two conditions were then proposed to the subjects after these 2 weeks of training. In the first one, the alpha male received a subanesthetic dose of ketamine $(0.3 \mathrm{mg} / \mathrm{kg}) 2 \mathrm{~min}$ prior to the daily 2 -h test session. The other 2 members of the group remained untreated. In the second condition, Roman received only a sham injection (control condition). Figure 9 represents the number of new stimulus pairs learned during $32 \mathrm{~min}$ postinjection - that is, when that low dose of ketamine had a detectable effect - as a function of test condition. Unsurprisingly, Roman was less efficient in learning under the influence of ketamine than after a sham injection. However, treatment of the alpha male had remarkable effects on the learning performance of the subordinate untreated female (Alison), who learned more pairs when Roman received a low dose of ketamine. This effect cannot be accounted for by greater trial frequencies for Alison when Roman was treated. In practice, Alison made fewer trials altogether in the ketamine condition (49.9 trials on average) than in the sham condition (70.4 trials), presumably because Roman stayed longer in front of the test system when treated. This finding demonstrates that the treatment applied to the alpha male released social pressures on the subordinate animal, although untreated, and promoted learning. It further demonstrates the power of ALDM to measure complex social influences on learning (Salun, 2006).

\section{DISCUSSION}

This article has presented a new setup for studying the motor, perceptual, or cognitive performance of monkeys in social groups and provides a set of preliminary data collected with that system. The first fact emerging from our research is the extremely high motivation of the monkeys to interact with the automatic test system, as evidenced by trial frequencies and the baboons' participation in the experiments at night. Thus, ALDM appears to be a very efficient strategy for collecting a large number of trials in a short period of time. The hierarchically organized structure of primate societies, including those of baboons, often prevents nondominant animals from accessing rare resources. If a favored food is given to the group, only the alpha male will have access to it (Itani, 1954). This dominance had little influence on trial frequencies in our task, except for a female under the strongest social pressure. In our research, shift of the subjects inside the test chamber was facilitated by continuous testing, providing ample opportunities to get around social constraints to interact with ALDM. It was

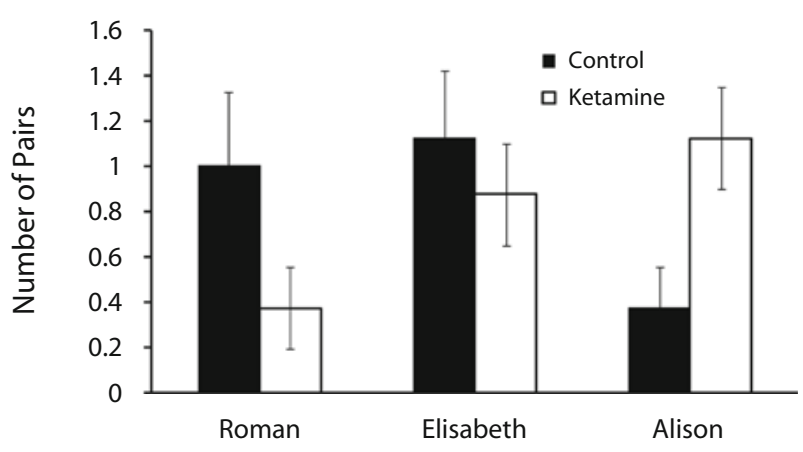

Figure 9. Performance (number of stimulus pairs learned and standard deviations) by subjects, depending on the treatment applied to Roman, the alpha male. 
also facilitated by the time-out introduced at the end of each test session. From this consideration, there are good reasons to believe that ALDM will work that efficiently with larger groups of monkeys, at least if the number of test systems is sufficiently large to limit social inhibitions.

Our second important result is that the monkeys were very proficient at learning during ALDM testing. Admittedly, the performance of our baboons must have been promoted by their past history in experimental research. Nevertheless, the naive monkeys tested in Study 3 demonstrated prompt learning with this system, since they learned a two-alternative forced choice task in approximately 2 weeks. Several aspects of our procedure likely account for fast and successful learning. First, the voluntary participation of the subjects may have promoted attention to the task. Second, although the monkeys remained in their group during testing, the design of the test chamber prevented the subject from seeing its partners when facing the screen and, therefore, limited external influences on learning. Finally, long bouts of interactions with ALDM must have favored short- and long-term memorization of the outcome of the behavior.

ALDM was developed to conduct studies on baboons' visual cognition, but the idea of automatically testing selfidentified animals is potentially of strong interest for a very large community of researchers. Thus, ALDM can be used to study a quasi-infinite number of questions in the domains of chronobiology, motor control, memory, perception, nonsocial cognition, and social cognition or with regard to the interaction between these domains. The data presented in Studies 1-3 or published in Fagot et al. (2008) confirm that the test system may be of interest for the study of a large spectrum of scientific questions. After adaptations, ALDM could also be employed with nonhuman primates other than baboons or macaques (e.g., chimpanzees, capuchins, etc.) and could even be used with nonprimate animals, such as birds or rodents. Moreover, ALDM is potentially of interest to biomedical researches - for instance, for assessing how motor or cognitive performance varies after brain lesions or pharmacological treatments (see Study 3). Finally, ALDM can be modified to study sensory modalities other than visionfor instance, if the touch screen is replaced by a tactile stimulation device.

In a different perspective, ALDM might also be considered as a tool for the enrichment of living conditions. ALDM combines two features that are known to promote well-being in primates: group testing (Griffin \& Harlow, 1966) and the possibility of having regular interactions with computers, which increases their daily activity (Washburn \& Rumbaugh, 1992). ALDM therefore also appears to be an interesting approach to promoting the well-being of animals in scientific research.

\section{AUTHOR NOTE}

D.P. developed the electronic components of ALDM, and J.F. contributed the other aspects of the methodology, computer programming, and research. Jean Philippe Logier is acknowledged for his help in designing the food dispenser. Thanks to Marc Martin and David Ohayon for their technical support. Olivier Blin, Joelle Micallef-Roll, and Thomas Salum are acknowledged for their contribution to Study 3. Correspondence concerning this article should be addressed to J. Fagot, LPC/CNRS/ UMR 6146, Pôle 3C, 3 place Victor Hugo, Bat 9, Case D, 13331 Marseille cedex 1, France (e-mail: joel.fagot@univ-provence.fr).

\section{REFERENCES}

Altman, S. A., \& Altman, J. (1971). Baboon ecology. Chicago: University of Chicago Press.

Andrews, M. W., \& Rosenblum, L. A. (1994). Automated recording of individual performance and hand preference during joystick-task acquisition in group-living bonnet macaques (Macaca radiata). Journal of Comparative Psychology, 108, 358-362.

DREA, C. M. (2006). Studying primate learning in group contexts: Tests of social foraging, response to novelty, and cooperative problem solving. Methods, 38, 162-177.

Drea, C. M., \& Wallen, K. (1999). Low-status monkeys "play dumb" when learning in mixed social groups. Proceedings of the National Academy of Sciences, 96, 12965-12969.

Evans, T. A., Beran, M. J., Chan, B., Klein, E. D., \& Menzel, C. R. (2008). An efficient computerized testing method for the capuchin monkey (Cebus apella): Adaptation of the LRC-CTS to a socially housed nonhuman primate species. Behavior Research Methods, 40, 590-596.

Fagot, J., Bonté, E., \& Parron, C. (2008). Concept of uprightness in baboons: Assessment with pictures of realistic scenes. Animal Cognition, 12, 369-379.

FAGOT, J., \& COOK, R. (2006). Evidence for large long-term memory capacities in baboons and pigeons and its implications for learning and the evolution of cognition. Proceedings of the National Academy of Sciences, 103, 17564-17567.

Fagot, J., \& Deruelle, C. (1997). Processing of global and local visual information and hemispherical specialization in humans (Homo sapiens) and baboons (Papio papio). Journal of Experimental Psychology: Human Perception \& Performance, 23, 429-442.

Fagot, J., Drea, C. M., \& Wallen, K. (1991). Asymmetrical hand use in rhesus monkeys (Macaca mulatta) in tactually and visually regulated tasks. Journal of Comparative Psychology, 105, 260-268.

FAGOT, J., \& PARRON, C. (2009). Relational matching in baboons (Papio papio) with reduced grouping requirements. Manuscript submitted for publication.

FITTS, P. M. (1954). The information capacity of the human motor system in controlling the amplitude of movement. Journal of Experimental Psychology, 47, 381-391.

Griffin, G. A., \& Harlow, H. F. (1966). Effects of three months of total social deprivation on social adjustment and learning in the rhesus monkey. Child Development, 37, 533-547.

ITANI, J. (1954). On the acquisition and propagation of a new food habit in the natural group of the Japanese monkeys at Takasakiyama. Primates, 1, 84-98.

Premack, D. (1983). The codes of beast and man. Behavioral \& Brain Sciences, 6, 125-167.

Rumbaugh, D. M. (1970). Learning skills of anthropoids. In L. A. Rosenblum (Ed.), Primate behavior: Developments in field and laboratory research (pp. 1-70). New York: Academic Press.

SALun, T. (2006). Effets sociaux d'un traitement subanesthésique de Kétamine chez un groupe de macaques rhésus (Macaca mulatta). Unpublished master's thesis, Université d'Aix-Marseille 2.

SILK, J. B. (1984). Inductive primatology: Primate social relationships. Science, 224, 981-982.

Thompson, R. K. R., Oden, D. L., \& Boysen, S. T. (1997). Languagenaive chimpanzees (Pan troglodytes) judge the relations between relations in a conceptual matching-to-sample task. Journal of Experimental Psychology: Animal Behavior Processes, 23, 31-43.

WASHBURN, D. A., \& RUMBaUgh, D. M. (1992). Investigations of rhesus monkey video-task performance: Evidence for enrichment. Contemporary Topics in Laboratory Animal Science, 31, 6-10.

(Manuscript received October 9, 2008; revision accepted for publication January 12, 2009.) 